\title{
Avances tecnológicos aplicados al estudio ergonómico de los puestos de trabajo
}

María Eugenia Figueroa Valenzuela

\begin{abstract}
- Resumen
Este artículo muestra la aplicación de tecnología de última generación, generando un sistema innovador para el análisis ergonómico de los puestos de trabajo.

Se explican las principales características de las investigaciones realizadas en este ámbito por el Laboratorio de Biomecánica y Salud Laboral de Mutual de Seguridad y apoyadas por un proyecto FONTEC- CORFO, que consiste en el desarrollo sistemas de análisis de movimiento y un programa computacional para su interpretación, además de un sistema de análisis de la fatiga muscular mediante electromiografía de superficie.
\end{abstract}

Palabras claves: Ergonomía, fatiga muscular, análisis de movimiento.

\begin{abstract}
- Abstract
This article shows to the application of technology of last generation, developing an innovating system for the ergonomic analysis of the workplaces.

The main characteristics of the investigations made at the Laboratorio de Biomecánica y Salud Laboral de Mutual de Seguridad and supported by a FONTEC- CORFO are explained. The proyect consist in developing systems of movement analysis and a computacional program for their interpretation, in addition an analysis system of the muscular fatigue by means of surface electromyography.
\end{abstract}

Key words: Ergonomics, mucular fatigue, movement analysis

Terapeuta Ocupacional, Licenciada en Terapia Ocupacional, Máster en Ergonomía. Profesor asistente de la Universidad de Chile. Encargada de la Unidad de Ergonomía del Laboratorio de Biomecánica y Salud Laboral de Mutual de Seguridad. Contacto > > Independencia 1027 


\section{- Introducción}

Este artículo muestra una experiencia desarrollada por el Laboratorio de Biomecánica y Salud Laboral de Mutual de Seguridad que cuenta un equipamiento de análisis biomecánico de alta tecnología, que desde el año 1998, mediante un proyecto de investigación FONTEC, co-financiado por CORFO y Mutual de Seguridad, ha ampliado el uso clínico de estos equipos a una aplicación en ergonomía, aplicable a la evaluación de los riesgos ergonómicos de puestos de trabajo.

En la primera etapa, correspondiente a un primer estudio, utilizando el equipamiento disponible en el Laboratorio para el análisis de movimiento, se generó un nuevo sistema de captación de éste aplicable a personas en movimiento libre, lo que se diferencia de los análisis convencionales aplicados en ergonomía que generalmente se realizan basados en análisis fijo de las posturas de trabajo y no consideran el factor de riego más relevante, el tiempo de exposición

En este estudio se integraron el análisis de movimiento y los parámetros de riesgo ergonómico más reconocidos por la literatura científica. Esta integración se logró mediante un programa computacional de desarrollo propio, que establece para cada segmento corporal un juicio de confort o riesgo en función del tiempo, contemplando la repetitividad y estaticidad.

Posteriormente, en un segundo estudio complementario, se estudia la fatiga muscular mediante la aplicación de mediciones de grupos musculares sometidos a esfuerzos con electromiografía de superficie. Ambas Investigaciones han permitido generar conocimientos sólidos que abren paso a la consolidación de un proyecto mayor, abriendo una nueva línea de trabajo para este Laboratorio, con el que se espera configurar una oferta de servicios para las empresas nacionales, que mediante las mejoras de las condiciones físicas de los puestos de trabajo, produzcan un aumento en la eficiencia productiva y mejoren las condiciones de trabajo.

\section{- El análisis de movimiento}

Es conocido que los métodos disponibles para la evaluación de los riesgos músculo esquelético como el RULA o el OWAS, se basan en el análisis de las diferentes posturas realizadas por un trabajador y se aplican en la evaluación de las condiciones físicas de los puestos de trabajo asociados al análisis de la actividad. De esta forma un Ergónomo observa qué posturas son las principales y determina el riesgo con los parámetros entregados por cada método.

De la observación deriva el primer inconveniente, ya que cada postura es analizada en forma estática, como una foto, y la actividad laboral es esencialmente dinámica. Es muy difícil encontrar puestos de trabajo donde los trabajadores permanezcan mucho tiempo en una misma posición o postura, especialmente si nos referimos a las extremidades superiores, que son los segmentos corporales que más presentan disfunciones. Por esta razón los análisis convencionales, basados en el estudio de la postura, son sólo una aproximación al problema ya que carecen de exactitud y requieren para su uso de profesionales con gran experiencia. 
Debido a lo anterior y a las facilidades derivadas de los avances tecnológicos, se desarrolló este innovador estudio centrado en el análisis de movimiento humano en la situación de trabajo.

Para el registro del movimiento se utiliz un analizador de movimiento CODA MPX30 que mediante captadores optoeléctricos (tridimensionales) y marcadores de superficie que cuantifican el movimiento articular con precisión. Este entrega los rangos articulares totales y arroja una gráfica de movimiento articular que es la "huella digital" del movimiento en estudio.

Fig. 1. Equipo de análisis de movimiento CODA: Scanner y captador optoeléctrico.
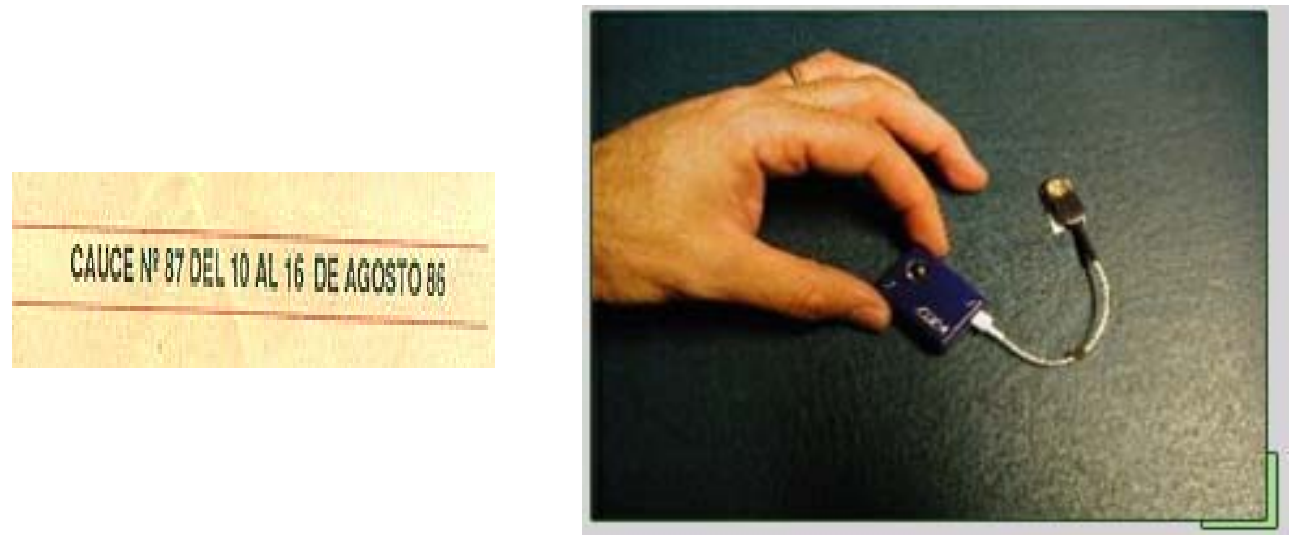

Para el análisis del movimiento se generó un programa computacional de análisis que permite establecer el tiempo que cada segmento corporal permanece en los distintos rangos de movimiento y la frecuencia con que esto sucede.

Uno de los requerimientos para la construcción del programa computacional, fue la construcción de modelos biomecánicos para los miembros superiores y columna, que determinan uno de los aspectos más relevantes de este estudio, dada su complejidad.

Una forma simplificada de comprender este sistema es el siguiente: los marcadores, que emiten una señal infrarroja se adhieren a la superficie de la piel en las prominencias óseas del sujeto en estudio, de esta forma los marcadores se muevan junto con las extremidades. Esta señal emitida es capturada por los scaners ( ver fig.1) y transmitida a un computador que analiza la posición en el espacio de cada marcador en todo momento, en base a las coordenadas (ejes $x, y, z$ ) en que se sitúan. El movimiento se representa mediante la unión de estos marcadores.

Esta representación inicial solo permite obtener los grados del movimiento según las coordenadas $(x, y, z)$ externas del entorno. Para que esta representación sea útil y los datos obtenidos representen los movimientos estudiados, es necesario construir un modelo biomecánico.

El modelo biomecánico ayuda a interpretar los datos capturados y entrega la información del movimiento de cada articulación en grados. La precisión de esta información fue de suma importancia para este estudio ya que un error en el diseño del modelo biomecánico, produciría una interpretación equivocada del movimiento y 
su comparación con los parámetros seleccionados para este estudio, no tendría validez.

Durante el proyecto se realizaron distintos modelos biomecánicos, con las herramientas del equipo de análisis de movimiento CODA. Inicialmente se consideró la confección de un modelo simple, sin embargo esto fue inaplicable dado que las condiciones de movimiento presentes en la actividad laboral, son muy diferentes a las utilizadas para el estudio en la clínica.

La complejidad del análisis en la actividad laboral esta determinada por la libertad de movimiento y los segmentos involucrados en ella. El movimiento de una articulación debe contemplar que muchas veces éste es producto del movimiento de otras articulaciones, por ejemplo el codo, es movilizado por el hombro. Entonces, para conocer el movimiento del codo es necesario construir un modelo que considere los posibles cambios en el espacio de éste, sin poder predecir la dirección del movimiento del sujeto.

Finalmente, los modelos biomecánicos fueron construidos con el apoyo de un experto el Sr. Phill Pikering, Ingeniero Matemático de la Empresa inglesa Charnwood Dynamics Limited, quien debió desarrollar complejos algoritmos matemáticos para lograr una captación válida. El programa computacional desarrollado permite detectar que segmento se encuentran en riesgo ergonómico, durante la ejecución de actividades complejas y junto con esto nos permite predecir el comportamiento del riesgo al modificar algunas condiciones del puesto de trabajo.

Fig.2 Resultado de la aplicación del análisis a un trabajador.

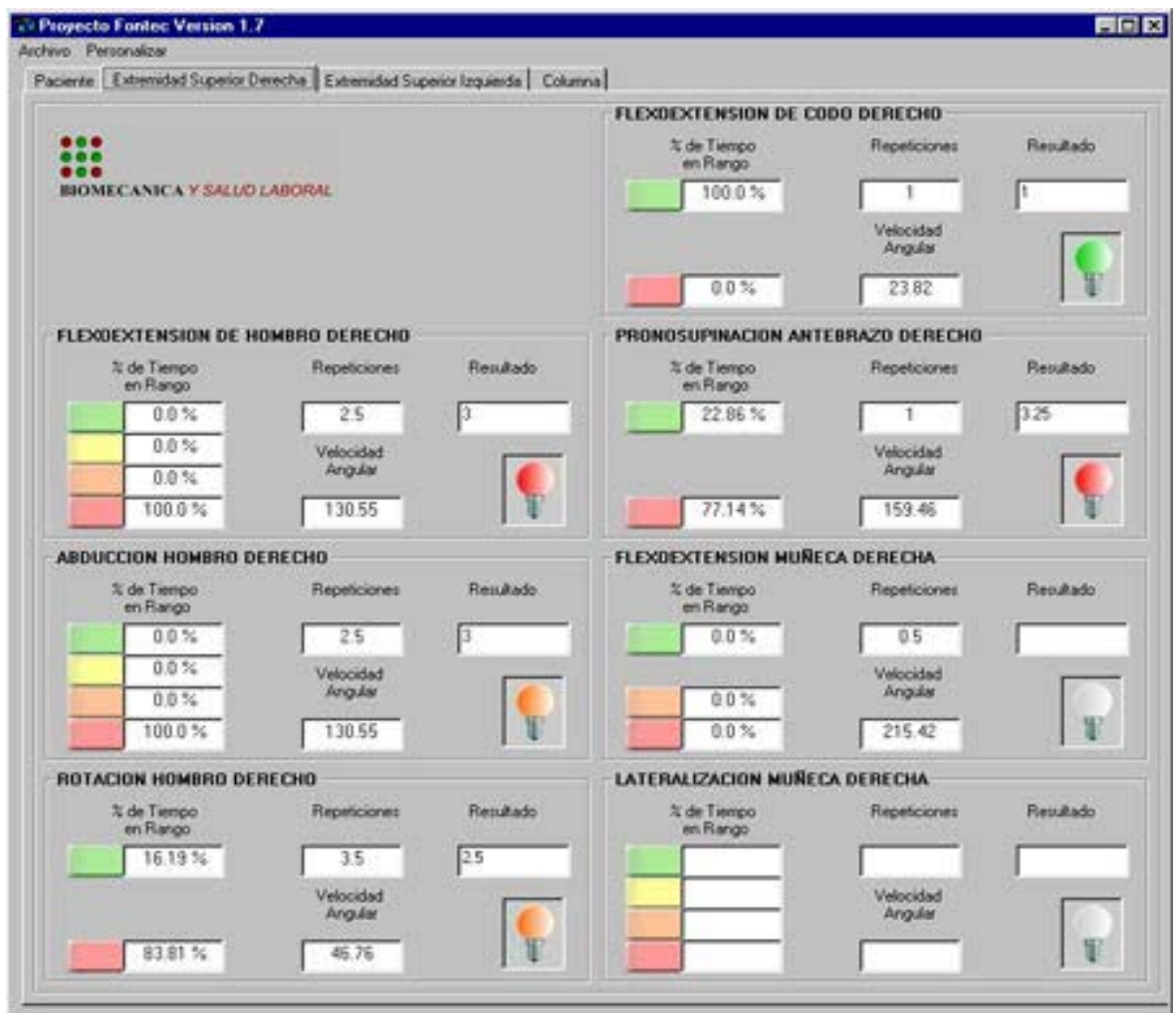




\section{El análisis de la fatiga muscular}

Este segundo estudio, que se centró particularmente en el análisis de la fuerza como factor de riesgo para la ocurrencia de desajustes músculo esqueléticos y es un complemento del estudio anterior.

La medida de la fuerza tiene una importancia básica en el estudio biomecánico del trabajo. En el campo de la ergonomía la distribución de esfuerzos y presiones del individuo en situación de trabajo, constituye uno de los criterios más importantes en la evaluación y diseño de puestos, útiles y herramientas de trabajo.

Desde una perspectiva puramente física y biomecánica, la carga o tensión que se genera en las diferentes articulaciones y en los tejidos blandos podría alcanzar fácilmente cientos de kilogramos. Sin embargo desde un punto de vista fisiológico, a medida que se incrementa el esfuerzo muscular, como consecuencia de cargas elevadas, disminuye la circulación sanguínea en el músculo pudiendo aparecer fatiga muscular. Cuando las exigencias físicas del trabajo sobrepasan las capacidades de los individuos, aparece la fatiga muscular, entendida como la disminución progresiva de la capacidad de seguir realizando trabajo del mismo nivel.

Existen distintas técnicas de evaluación del esfuerzo desarrollado al realizar una tarea. Algunas miden directamente la carga manipulada por el trabajador o los esfuerzos que se producen en la interfase trabajador - entorno de trabajo; otras técnicas tratan de estimar el esfuerzo muscular realizado durante la tarea midiendo la actividad eléctrica de los músculos que realizan un mayor esfuerzo; otras se basan en medidas de aceleración de los segmentos corporales; etc.

En la actualidad existen muchas aproximaciones y niveles de sofisticación para medir las fuerzas desarrolladas al realizar un trabajo. En este trabajo se analizó la técnica de la electromiografía (EMG) de superficie por corresponder a la técnica utilizada en el Laboratorio de Biomecánica de Mutual de Seguridad, quien auspició esta investigación.

La electromiografía permite evaluar la actividad muscular desarrollada por un trabajador en un determinado grupo muscular, durante la realización de la tarea. Es una herramienta valorada como aplicación ergonómica si se utiliza correctamente y se aprecian también sus limitaciones. La EMG puede ser aplicada como un método analítico pero debe ser interpretado con la interpretación de un experto.

Las pruebas realizadas en este estudio nos permitieron valorar el comportamiento de un mismo grupo muscular en variadas posiciones, permitiendo tener una imagen clara de la actividad muscular bajo distintas condiciones de diseño del puesto de trabajo y a la vez, generar una retroalimentación para el rediseño de la estación de trabajo, contribuyendo también a la generación de modelos biomecánicos. 


\section{- Un ejemplo de aplicación}

Los conocimientos y técnicas generadas en las investigaciones señaladas se aplicaron en el estudio del puesto de trabajo de "camarógrafo de televisión", quienes utilizan un soporte para las cámaras llamado Steadicam.

El trabajo de camarógrafo se realiza sometido a diferentes exigencias de orden ambiental (ruido excesivo, temperatura fluctuante, iluminación variable y riesgo por caídas), mental (elevados niveles de atención y concentración, requerimiento de respuestas inmediatas, coordinación visomotriz y escaso nivel de autonomía) que acentúan la carga física, dónde se centra nuestro estudio mediante la aplicación biomecánica.

Fig. 3 Camarógrafo con Steadicam

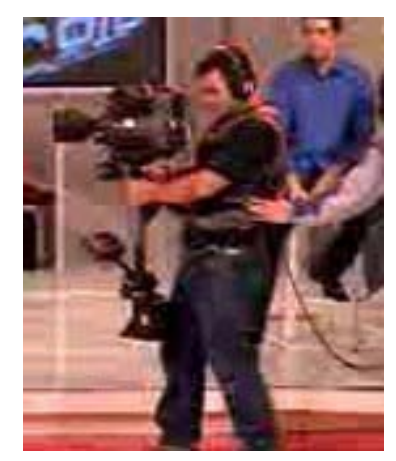

Otros antecedentes significativos son: el peso soportado es variable dependiendo de la cámara utilizada y fluctúa ente 20 y 25 Kilos, lo que genera un aumento considerable en el gasto energético y el diseño de la Steadicam que desplaza el centro de gravedad hacia adelante, generando un aumento en el brazo de palanca incrementando la carga lumbar.

La movilidad del brazo, genera que la ubicación del centro de gravedad del equipo sea variable en cada instante, exigiendo distintos niveles de actividad (contracciónrelajación) de la musculatura del tronco.

En el análisis más detallado de las exigencias físicas y del estudio biomecánico se detalla a continuación. 


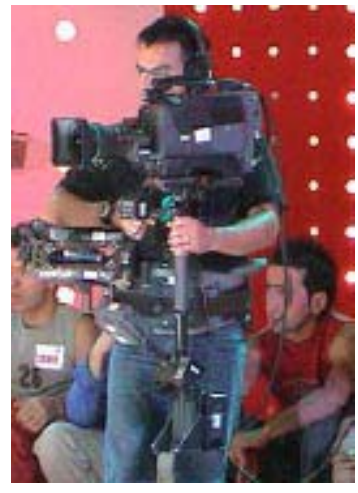

\section{Estáticas}
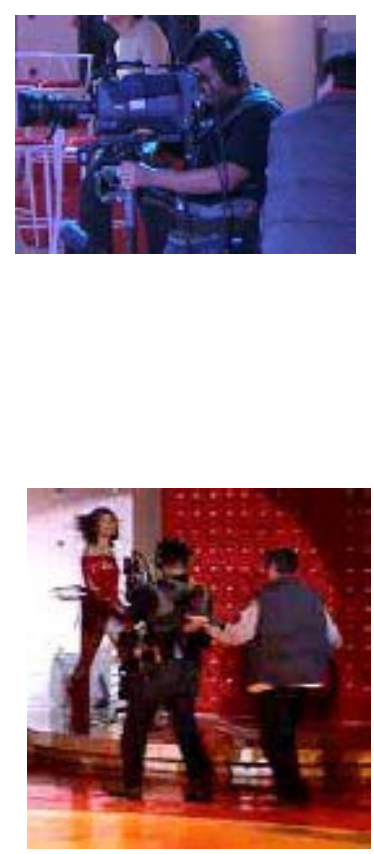

Dinámicas

\section{Posturas asimétricas:}

Las condiciones de ejecución de la actividad implican que el operador permanezca con la carga (equipo) fuera de la línea media del cuerpo. Dicha condición, provoca una distribución asimétrica de los pesos, generando cambios eléctricos diferentes a cada lado de la musculatura paravertebral.

\section{Posturas mantenidas:}

Durante toda la tarea el cuello permanece en flexión de $40^{\circ}$ aprox., generando una contracción sostenida de los músculos flexores y extensores, que podría generar molestias en la zona

\section{Giros:}

Los requerimientos de la tarea obligan a realizar movimientos de rotación de tronco, sobrecargando las estructuras de soporte de la columna, favoreciendo la aparición de enfermedades de los discos intervertebrales.

\section{Cambios de aceleración:}

El manejo del equipo requiere constantes cambios de aceleración que solicitan, fisiológicamente, un gran nivel de coordinación muscular que aumenta el nivel de fatiga $y$ favorece la aparición de lesiones.

Fig.4 - 5 - 6 Observación de las exigencias físicas de la tarea en terreno 
Se reprodujeron y analizaron en el Laboratorio las exigencias más relevantes detectadas mediante la observación directa de la ejecución de la actividad, con la finalidad de cuantificar el trabajo muscular a nivel lumbar y determinar el riesgo a que están sujetos estos trabajadores utilizando los parámetros disponibles en la literatura científica

Se utilizó la medición electriomiográfica, la que permite valorar los cambios eléctricos musculares generados en las distintas condiciones de actividad presentes en la tarea de camarógrafo.

Previo a la determinación de los niveles de actividad se evaluó la musculatura lumbar alta y baja del trabajador en condiciones de reposo y de máxima capacidad.

\section{Condiciones evaluadas}

\section{Bipedestación.}

Con carga externa/ cámara y accesorios con un peso total de 21 kilos.

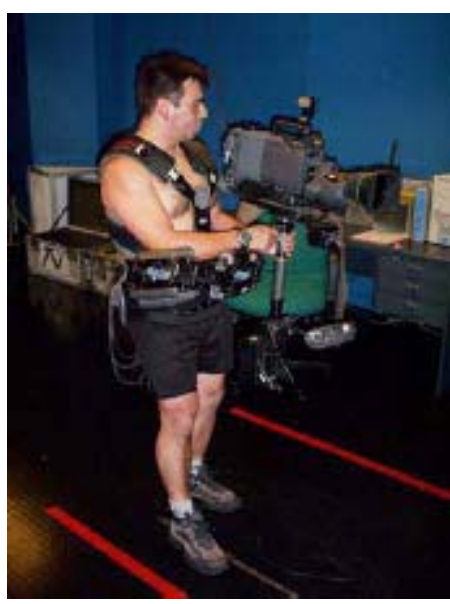

\section{Actividad eléctrica}

\section{Derecho}

\section{Izquierdo}

\section{Alta}

249

157

Baja

270

201

\section{Nivel \\ de riesgo}
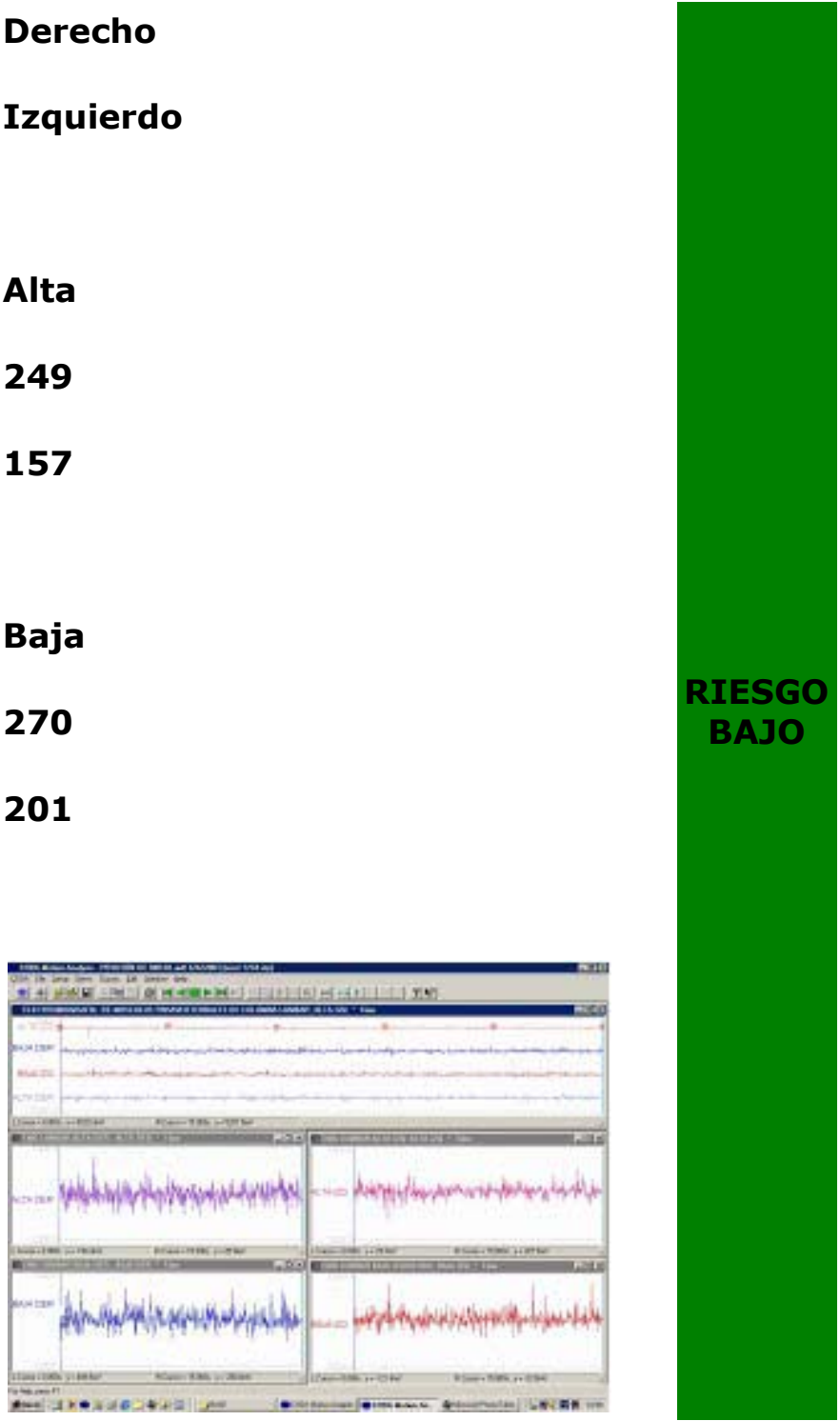
2. Marcha. Con carga externa

Se registra la actividad eléctrica mientras el individuo camina tranquilamente, simulando la filmación.

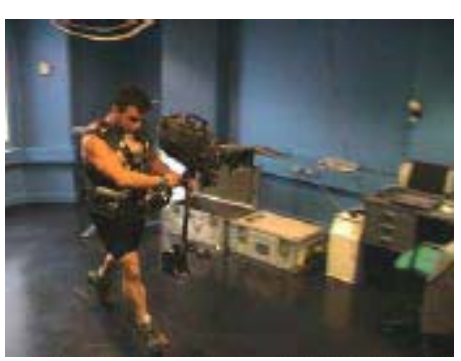

\section{Trote con carga externa}

Se registra la actividad eléctrica mientras el individuo trota simulando la filmación.

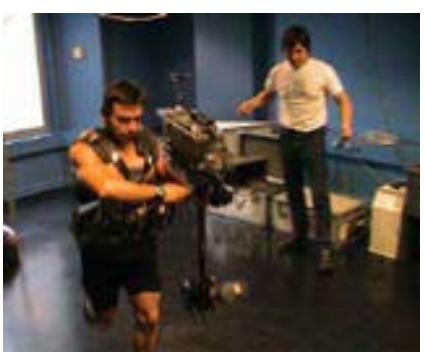

Derecho

\section{Izquierdo}

Alta

$410(28.6 \%) *$

$421(28.6 \%) *$

Baja

$420(34.9 \%) *$

$390(34.1 \%) *$

RIESGO

MEDIO

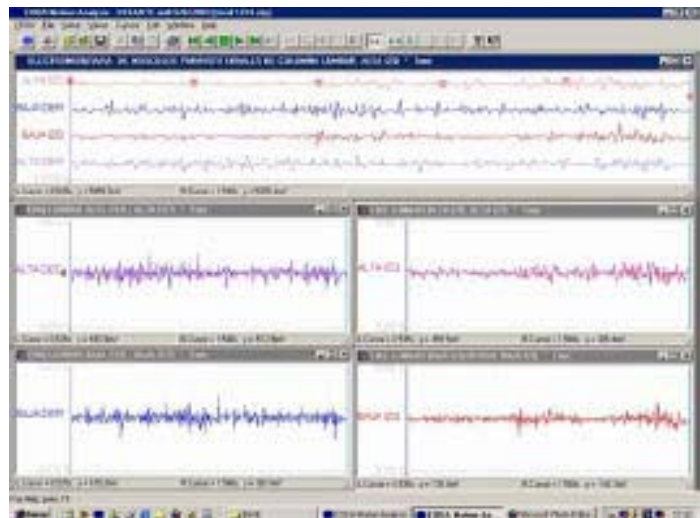

Derecho

\section{Izquierdo}

Alta

917 (64\%)*

769 (52.31\%)*

Baja

$834(69.4 \%) *$

$931(81.5 \%) *$ 


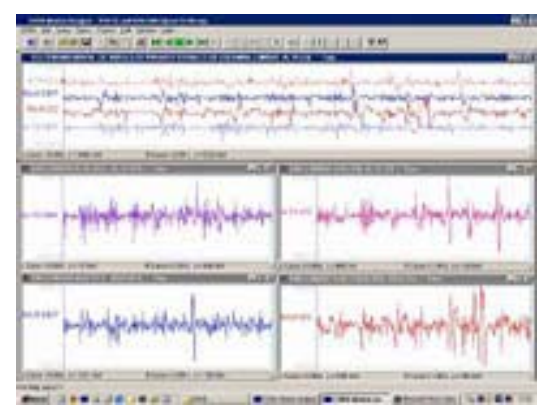

\section{Carrera con carga externa}

Se registra la actividad eléctrica mientras el individuo corre simulando estar filmando.

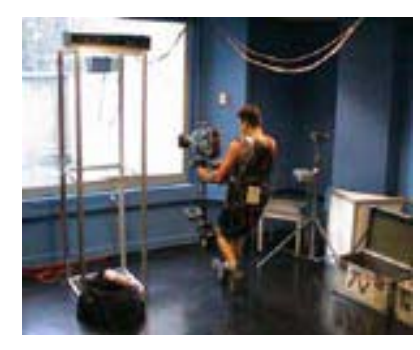

\section{Derecho}

\section{Izquierdo}

\section{Alta}

$845(58.6 \%) *$

$940(63.9 \%) *$

\section{Baja}

1095 (91.1\%)*

$1122(98.2 \%) *$

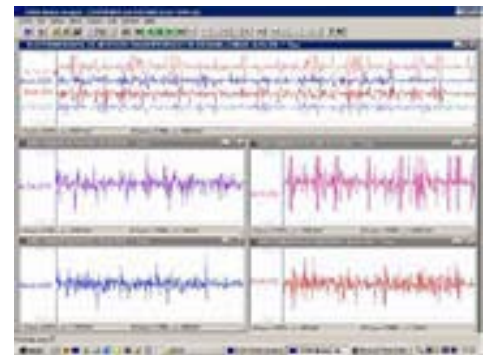

Derecho

\section{Izquierdo}

Se registra la actividad eléctrica mientras el individuo reproduce el gesto de subir peldaños. Alta

$376(26.4 \%) *$

$344(24.3 \%)^{*}$

Baja

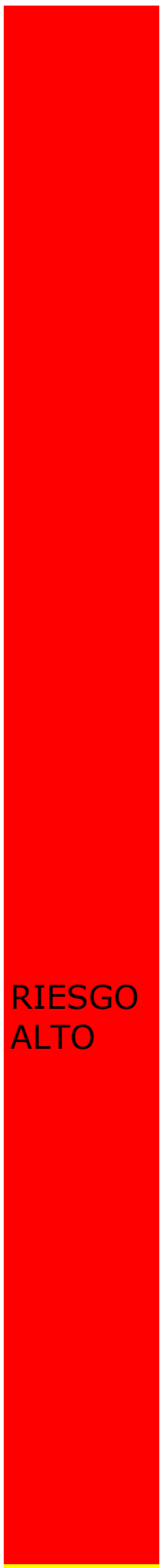

RIESGO MEDIO 


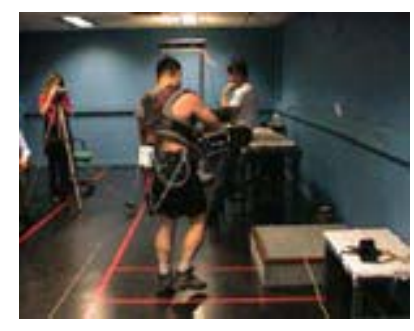

$$
\begin{aligned}
& 422(35.1 \%)^{*} \\
& 436(38.1 \%)^{*}
\end{aligned}
$$

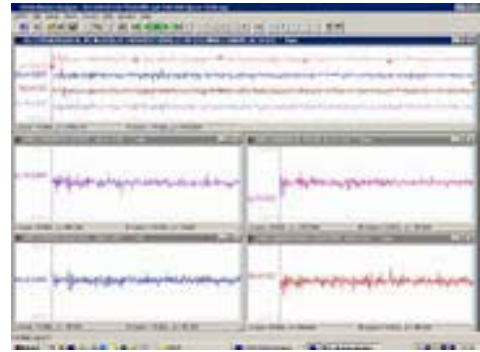

\section{* de la contracción máxima voluntaria (CMV)}

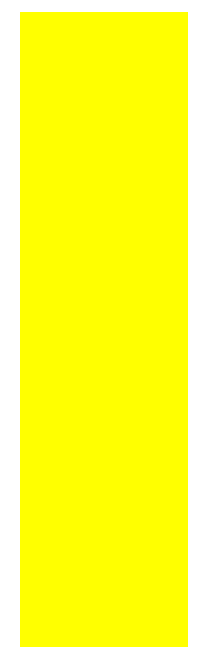

Figs. 7 -8-9-10-11 Pruebas en laboratorio y señal Electromiográfíca de las mismas.

Este estudio permitió demostrar que el puesto de trabajo de camarógrafo, utilizando el soporte steadicam representa una gran exigencia para la musculatura lumbar, especialmente en las operaciones donde debe desplazarse con la cámara. Esta demanda muscular expone al trabajador al riesgo de lesiones tanto en la musculatura lumbar como en la columna vertebral. Estos datos nos permitieron entregar recomendaciones fundadas en datos objetivos tanto a la empresa como a los trabajadores.

\section{- Conclusión}

El análisis de movimiento con este sistema permite cuantificar las tareas realizadas por el trabajador en lo relativo al riesgo postural y de movimiento. Al ser ejecutadas en tiempo real e incorporar el tiempo de exposición, lo diferencia de los métodos tradicionales, convirtiéndolo en una innovadora herramienta que entrega información de gran valor para adaptar los puestos de trabajo a las capacidades de las personas.

La medida de la fuerza tiene una importancia básica en el estudio biomecánico del trabajo. En el campo de la ergonomía la distribución de esfuerzos y presiones del individuo en situación de trabajo, constituye uno de los criterios más importantes en la evaluación y diseño de puestos, útiles y herramientas de trabajo. Mediante las pruebas realizadas en este estudio se permitió valorar el comportamiento de distintos grupos musculares frente a exigencias variadas, permitiendo comprender la fatiga segmentaría responsable de molestias y enfermedades cuando se sostiene en el tiempo

Ambos estudios demuestran las grandes posibilidades que ofrece el desarrollo tecnológico aplicado a la disciplina de la Ergonomía. Siempre es útil contar con herramientas que permitan cuantificar los esfuerzos, ya que de esta forma nos acercamos a establecer parámetros claros y objetivos en cuanto a las limitaciones 
humanas, que serán un paso para controlar las exigencias a que están sometidos algunos trabajadores.

\section{- Bibliografía}

1. FIGUEROA,ME (2001) "El análisis del movimiento aplicado al estudio ergonómico de los puestos de trabajo". Informe de Proyecto FONTEC

2. FIGUEROA,ME; BELMAR,R (2002) "El análisis de la fuerza como factor de riesgo" Investigación Laboratorio de Biomecanica y Salud Laboral de Mutual de Seguridad.

3. FIGUEROA,ME ; BELMAR,R (2002) "Estudio biomecánico del puesto de camarógrafos de steadican de TVN"

4. www.biomecanica.cl. Página web del Laboratorio de Biomecanica y Salud Laboral de Mutual de Seguridad. 